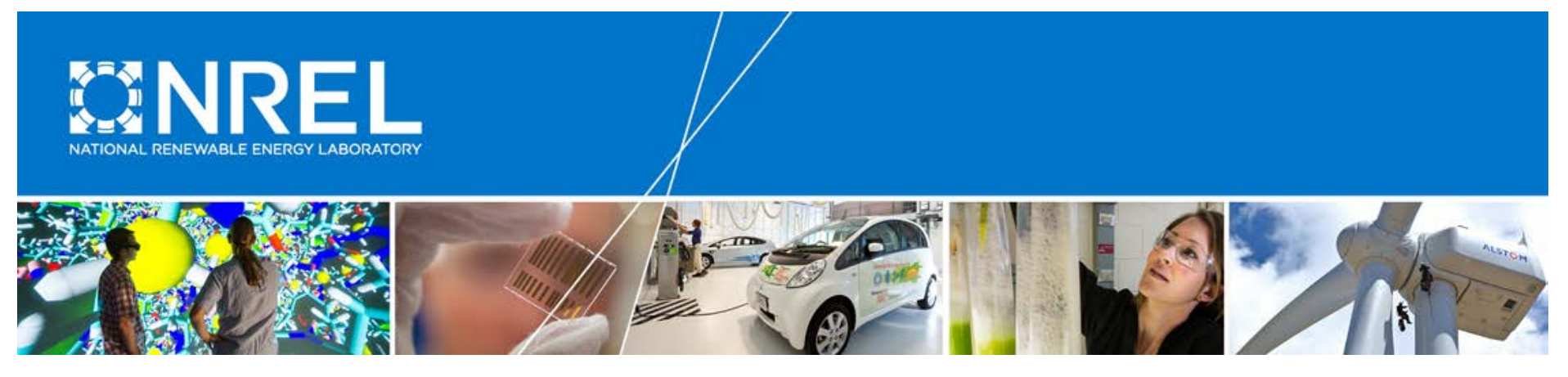

\title{
Turbine Control of a Tidal and River Power Generator
}

\section{Preprint}

Eduard Muljadi, Vahan Gevorgian, and Alan Wright

National Renewable Energy Laboratory

James Donegan, Cian Marnagh, and Jarlath McEntee

Ocean Renewable Power Company

To be presented at the 2016 IEEE North American Power

Symposium

Denver, Colorado

September 18-20, 2016

(C) 2016 IEEE. Personal use of this material is permitted. Permission from IEEE must be obtained for all other uses, in any current or future media, including reprinting/republishing this material for advertising or promotional purposes, creating new collective works, for resale or redistribution to servers or lists, or reuse of any copyrighted component of this work in other works.

NREL is a national laboratory of the U.S. Department of Energy Office of Energy Efficiency \& Renewable Energy Operated by the Alliance for Sustainable Energy, LLC

This report is available at no cost from the National Renewable Energy Laboratory (NREL) at www.nrel.gov/publications.

\section{Conference Paper}

NREL/CP-5D00-66867

August 2016

Contract No. DE-AC36-08G028308 


\section{NOTICE}

The submitted manuscript has been offered by an employee of the Alliance for Sustainable Energy, LLC (Alliance), a contractor of the US Government under Contract No. DE-AC36-08GO28308. Accordingly, the US Government and Alliance retain a nonexclusive royalty-free license to publish or reproduce the published form of this contribution, or allow others to do so, for US Government purposes.

This report was prepared as an account of work sponsored by an agency of the United States government. Neither the United States government nor any agency thereof, nor any of their employees, makes any warranty, express or implied, or assumes any legal liability or responsibility for the accuracy, completeness, or usefulness of any information, apparatus, product, or process disclosed, or represents that its use would not infringe privately owned rights. Reference herein to any specific commercial product, process, or service by trade name, trademark, manufacturer, or otherwise does not necessarily constitute or imply its endorsement, recommendation, or favoring by the United States government or any agency thereof. The views and opinions of authors expressed herein do not necessarily state or reflect those of the United States government or any agency thereof.

This report is available at no cost from the National Renewable Energy Laboratory (NREL) at www.nrel.gov/publications.

Available electronically at SciTech Connect http:/www.osti.gov/scitech

Available for a processing fee to U.S. Department of Energy and its contractors, in paper, from:

U.S. Department of Energy

Office of Scientific and Technical Information

P.O. Box 62

Oak Ridge, TN 37831-0062

OSTI http://www.osti.gov

Phone: 865.576.8401

Fax: 865.576.5728

Email: reports@osti.gov

Available for sale to the public, in paper, from:

U.S. Department of Commerce

National Technical Information Service

5301 Shawnee Road

Alexandria, VA 22312

NTIS http://www.ntis.gov

Phone: 800.553 .6847 or 703.605 .6000

Fax: 703.605.6900

Email: orders@ntis.gov 


\title{
Turbine Control of a Tidal and River Power Generator
}

\author{
E. Muljadi, A. Wright, V. Gevorgian, J. Donegan, C. Marnagh, J. McEntee
}

\begin{abstract}
As renewable generation has become less expensive during recent decades, and it becomes more accepted by the global population, the focus on renewable generation has expanded to include new types with promising future applications, such as river and tidal generation. The input variations to these types of resources are slower but also steadier than wind or solar generation. The level of water turbulent flow may vary from one place to another, however, the control algorithm can be adjusted to local environment.

This paper describes the hydrokinetic aspects of river and tidal generation based on a river and tidal generator. Although the information given in this paper is not that of an exact generator deployed on site, the data used is representative of a typical river or tidal generator. In this paper, the hydrokinetic and associated electrical controller of the system were not included; however, the focus of this paper is on the hydrodynamic control.
\end{abstract}

Index Terms - ocean power, marine, hydrokinetic, tidal, river, renewable, water power, variable generation, distributed generation.

\section{INTRODUCTION}

$\mathrm{T}$ HIS paper is based on the hydrokinetic power generating system for a river and tidal generator. The analysis is based on a tentative river generator with the power rating of $40 \mathrm{~kW}$. This paper focuses on the hydrokinetic power conversion, and the electrical system is simplified to clarify the hydrokinetic system and its associated control system.

Renewable energy continued to grow in 2014 against a backdrop of increasing global energy consumption and a dramatic decline in oil prices during the second half of the year. Hydropower is considered one of the most important renewable energy sources. Renewable energy sources are the second largest contributor to global electricity production, after fossil fuel.

Two types of tidal energy conversion use water flow to drive the turbine generator: one is based on utilizing the difference between the high and low tides, and the other is based on utilizing the water flow during the flood and ebb of the water. Tidal generation is more predictable than wind or solar generation; however, the level of turbulence and the impacts on the conversion device are no less severe than they are with wind turbulence [1].

Prospecting tidal energy is no trivial matter. Monitoring, measuring, and performing numerical simulations are used to compute the potential energy that can be harvested in a regional area. Various numerical methods are used to forecast the annual energy production at different sites to ensure successful tidal deployment [2-3].

Marine hydrokinetic energy resources have very promising futures. Various types of marine hydrokinetic generators have been explored, and some of have been commercialized. [4-5].

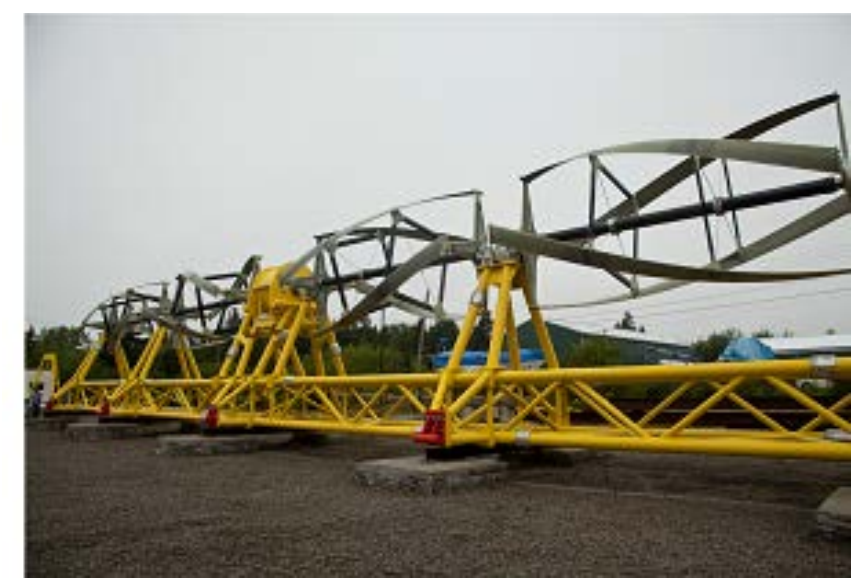

Fig. 1. Typical configuration of a river and tidal generator

A typical tidal generator can be mounted on the bottom of an estuary, and its shape and structure are similar to that of a Savonius wind turbine generator. It may be placed vertically or horizontally depending on the resource and the site.

This paper is arranged as follows. Section II describes the hydrodynamic power conversion, followed by the dynamic simulation in Section III. Finally, Section IV presents the conclusion.

\section{HYDRODYNAMIC POWER CONVERSION}

The technology used in tidal energy conversion is based on past work in designing propellers and the blades found on wind and hydropower turbines. The equations used to compute the energy conversion are very similar to the equations used in wind energy conversion.

The hydrodynamic power conversion in this type of generation is very similar to the generating system of wind power generation. It consists of major components: (a) the prime mover - the water turbine driving the generator, and (b) the electrical power conversion - the conversion from the output power of the generator at variable frequency into the power injected into the grid. This paper focuses on the turbine control using electrical power to position the turbine's operating point on the hydrodynamic characteristic of the water turbine.

\section{A. Hydrodynamic Characteristic}

The typical performance coefficient of the turbine is shown in Figure 2. This type of $C_{p}$ characteristics has a major advantage in that the turbine can be stalled to control the 
rotational speed and thus preventing a runaway condition from occurring without the need for pitch control as in a wind turbine.

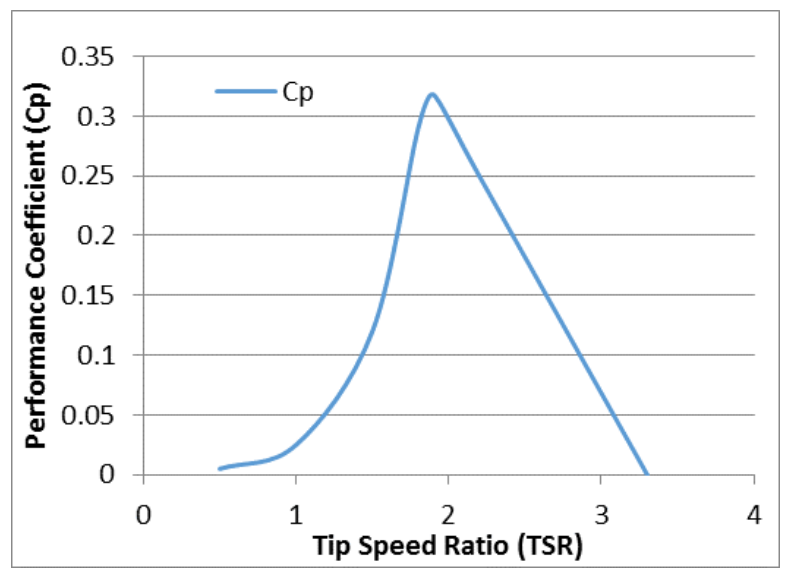

Fig. 2. Typical characteristic of a tidal turbine system

The available hydrokinetic power of a water turbine can be computed from the water flow and the turbine dimension as

$$
P_{\text {turbine }}=0.5 \rho A C_{p} V^{3}
$$

Where $\mathrm{R}$ is the radius of the turbine, $\mathrm{A}$ is the area of the turbine, $\rho$ is the water density, $\mathrm{V}$ is the water flow, and $\mathrm{C}_{\mathrm{p}}$ is the performance coefficient of the turbine.

The tip speed ratio (TSR) is defined as the ratio of the linear speed of the tip of the blade to the water speed.

$$
T S R=\frac{\omega R}{V}
$$

where the rotational speed, $\omega$, is the rotational speed of the blade. As shown in Figure 3, there is a specific TSR that has a maximum operating $\mathrm{C}_{\mathrm{p}}$.

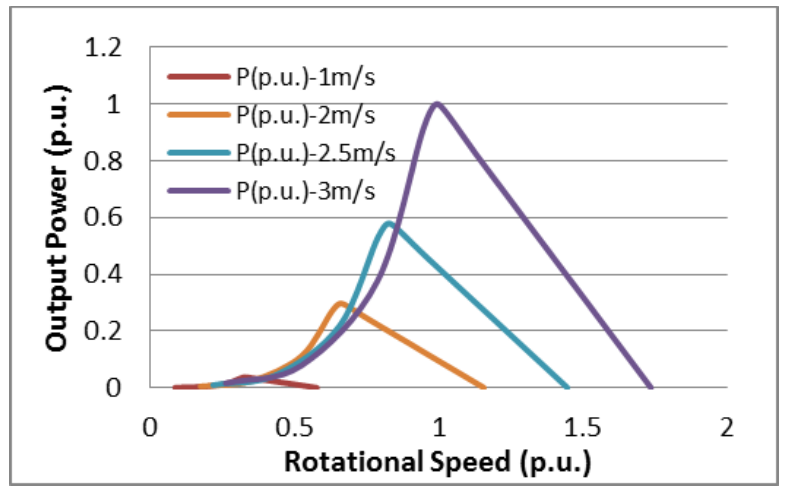

Fig. 3. Set of power characteristics of a tidal turbine system

The water flow, $\mathrm{V}$, in equation (1) can be substituted by the rotational speed, $\omega$, in equation (2). The new equation can be written as

$$
P_{\text {turbine }}=0.5 \rho A C_{p}\left(\frac{\omega R}{T S R}\right)^{3}
$$

The corresponding power-versus-speed characteristic for different flow speeds is given in Figure 3.

\section{B. Maximum Power Point Tracking}

Many maximum power point tracking systems with active search solutions have been developed during the years [5-6]. In this paper, we offer a passive control by using the rotor speed to guide the operating point of the tidal generator. This concept has been used successfully in wind power generation, and it provides a simple but stable operation. By substitution, we can replace $C_{p}$ with $C_{p \max }$ and the TSR with TSR $\mathrm{Tpmax}_{\text {. The }}$ resulting equation (3) can be used to control the generator and power converter as shown in equation (4).

$$
P_{\text {gen }}=0.5 \rho A C_{\text {pmax }}\left(\frac{\omega R}{T S R_{C p \max }}\right)^{3}
$$

The equation used to control the generation can be further simplified as in equation (5).

$$
P_{\text {genCpmax }}=K_{\text {Cpmax }} \omega^{3}
$$

where

$$
K_{C \max }=0.5 \rho A C_{\text {pmax }}\left(\frac{R}{T S R_{C p \max }}\right)^{3}
$$

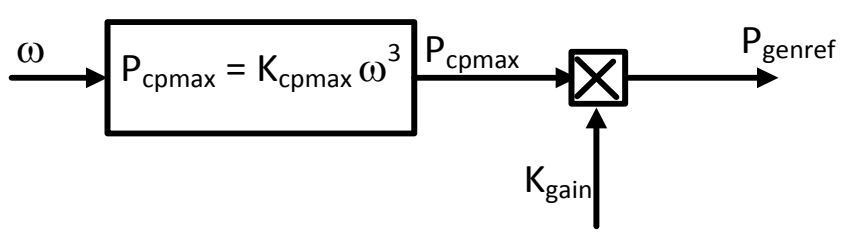

Fig. 4. Typical power control of a tidal turbine system

Figure 4 shows the implementation of the feed-forward control by controlling the generator output as a cube function of the rotational speed. This type of control is often used in variable-speed wind turbine generation and has been proven successful. The control algorithm can be implemented by using a prescribed power-speed lookup table as shown in equations (4-5). Note that under normal circumstances, the value of $\mathrm{K}_{\text {gain }}$ is constant at one per unit. As shown later, this value can be adjusted on the fly.

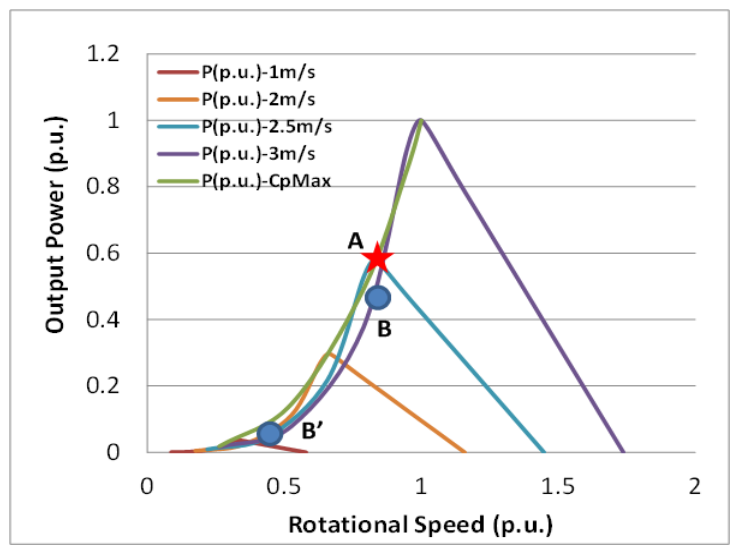

Fig. 4. Typical power control of a tidal turbine system 
Figure 4 shows the hydrokinetic power of the turbine as lines with symbols in different colors to represent different water flow speeds. The electrical output power is commanded (by controlling the power converter connected to the generator) to follow the equation, which is the cube function of the rotational speed (5), and it is shown as the green line in Figure 4. Unfortunately, this controller may lead to an unstable operating point. To illustrate the unstable operation, assume that the turbine originally operates at a water flow of $2.5 \mathrm{~m} / \mathrm{s}$ corresponding to Point $\mathrm{A}$. The generator power is commanded to follow the $\mathrm{C}_{\text {pmax }}$ (green line), and the hydrokinetic power corresponds to the blue line. A sudden increase of the water flow from $2.5 \mathrm{~m} / \mathrm{s}$ to $3 \mathrm{~m} / \mathrm{s}$ will not bring the hydrokinetic power up; instead, it will bring the hydrokinetic power down. As shown in Figure 4, the hydrokinetic power originally coincides with the generator output power at Point A (star). As the flow speed increases to $3 \mathrm{~m} / \mathrm{s}$, the hydrokinetic power suddenly drops from Point A to Point B. As a result, the output of the generator is higher than the hydrokinetic power; thus, the rotational speed slows down. The operating point of the hydrokinetic power will follow the purple curve (for a water flow of $3 \mathrm{~m} / \mathrm{s}$ ) from Point B to Point B', the generator power will follow the green curve from Point A to Point B', and the generator power will meet the hydrokinetic power at Point B', the new stable operating point.

\section{DYNAMIC SIMULATION}

In this section, the dynamic simulations are performed to verify the steady-state prediction and observe the dynamic behavior of the tidal turbine system under variations of water flow.

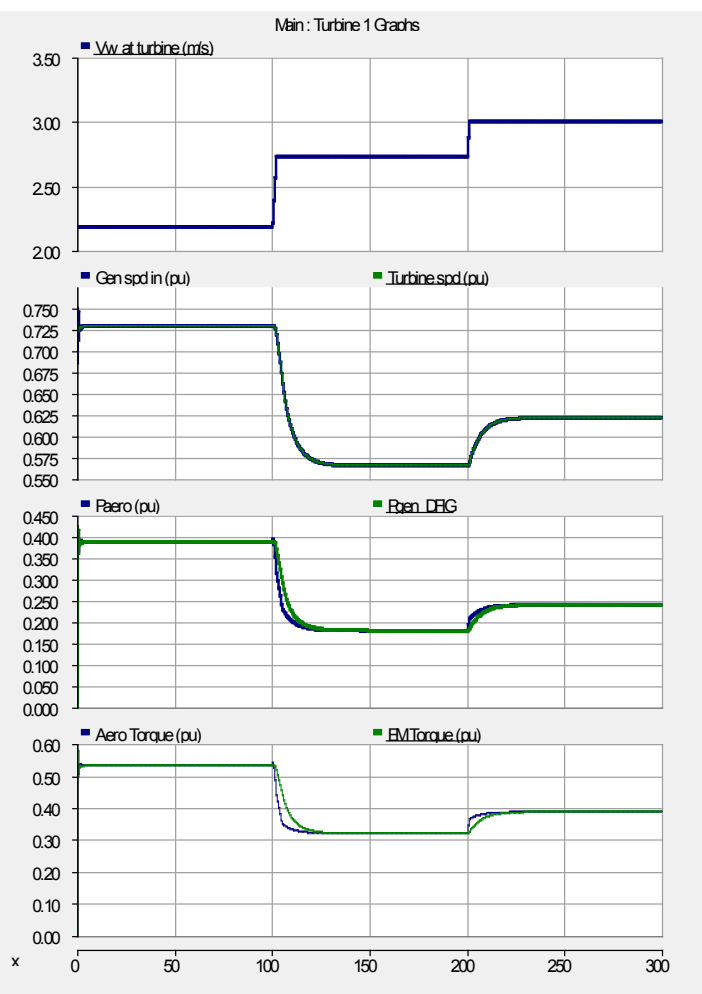

Fig. 5. Dynamic simulation to illustrate possible instability of the operation.
Changes to the control algorithms are proposed and simulated to verify the performance and observe the stability and improvement in the energy capture of the turbine system.

Figure 5 shows the operation of the turbine at $\mathrm{C}_{\mathrm{pmax}}$ based on equation (5) to illustrate the unstable operation. The water flow was originally at $2.2 \mathrm{~m} / \mathrm{s}$, it increases at $2.75 \mathrm{~m} / \mathrm{s}$, and instantaneously the operating point moves to the left of the $\mathrm{TSR}_{\text {tgt }}$. The hydrokinetic power of the turbine becomes lower than the electrical output power, thus slowing the rotational speed of the turbines, and the TSR becomes even smaller. Further increasing the water flow to $3 \mathrm{~m} / \mathrm{s}$ does not improve the $\mathrm{C}_{\text {pmax }}$.

The corresponding TSR and the operating $\mathrm{C}_{\mathrm{p}}$ can be illustrated in Figure 6. This operation becomes unstable when the turbine is entering the stall region where the performance coefficient, $\mathrm{C}_{\mathrm{p}}$, collapses as the TSR drops significantly.

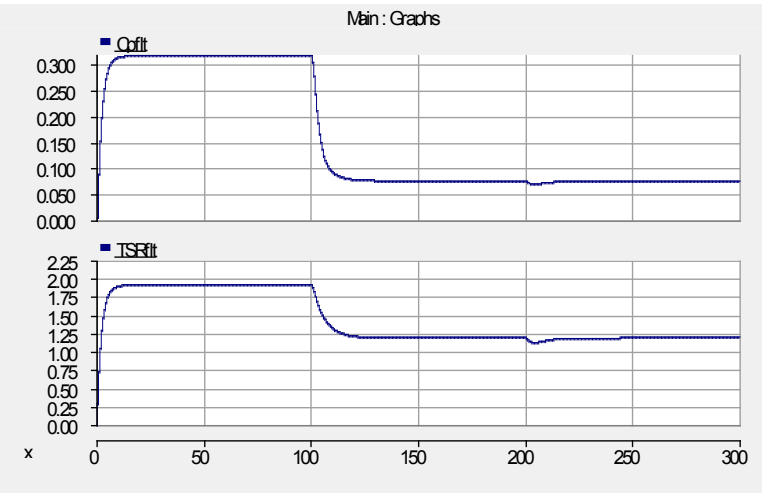

Fig. 6. Operating $\mathrm{C}_{\mathrm{p}}$ and the TSR as the water flow changes in an unstable operation

\section{A. Modified Power-Speed Command to Improve Stability}

The controller modified in the previous section can be further improved by changing the power controller when the water flow is in the medium to high range (between $2.5 \mathrm{~m} / \mathrm{s}$ and $3 \mathrm{~m} / \mathrm{s}$ ). As such, a better energy yield can be achieved, but the system is more stable in the low to medium water flow. The reasoning behind this control strategy is that in the higher water flow we want to allow the system to stall to ensure that a runaway situation will not occur.

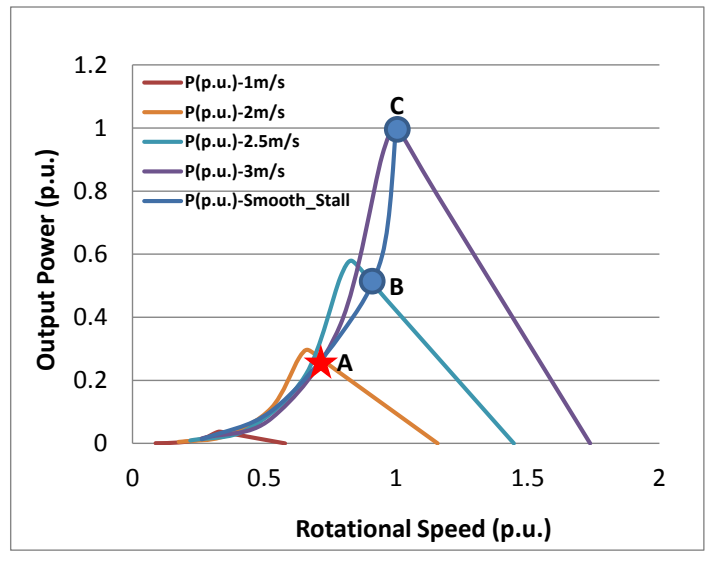

Figure 7. Hydrokinetic power and the generator power operated at $\mathrm{C}_{\mathrm{p}_{\_} \text {adjusted }}$ in low water flow $(0-2.5 \mathrm{~m} / \mathrm{s})$ and changed to $\mathrm{C}_{\mathrm{p}_{-} \max }$ in higher water flow $(>2.5$ $\mathrm{m} / \mathrm{s}$ ) as a function of the rotational speeds 
Figure 7 shows the modified power-speed path between Point B and Point C. As shown, the slope from Point B to Point $\mathrm{C}$ increases. This change can be expressed in equations (6-9). Note that this change can be made more aggressive to force-stall the turbine in the higher water flow regions by changing the value of $K_{\text {slope }}$ expressed in (7). Stalling the turbine in higher water flow will ensure that the turbine does not experience a runaway condition when a sudden change in water flow increases above the rated flow. Thus, without a pitching mechanism, this control strategy can be adopted.

The operation can be illustrated by referring to the power performance characteristics shown in Figure 8, wherein the operating point between the low and medium water flow is slightly less than the peak $\mathrm{C}_{\mathrm{p}}\left(\mathrm{C}_{\mathrm{p}_{-} \max }\right)$. Thus, from low to medium water flow, the generator will be operated to stay at $\mathrm{C}_{\mathrm{p}_{\mathrm{a} a d j u s t e d}}$ for stability purposes. From medium to high water flow, the turbine will be transitioned from $\mathrm{C}_{\mathrm{p}_{-} \text {adjusted }}$ to $\mathrm{C}_{\mathrm{p}_{-} \max }$ operation.

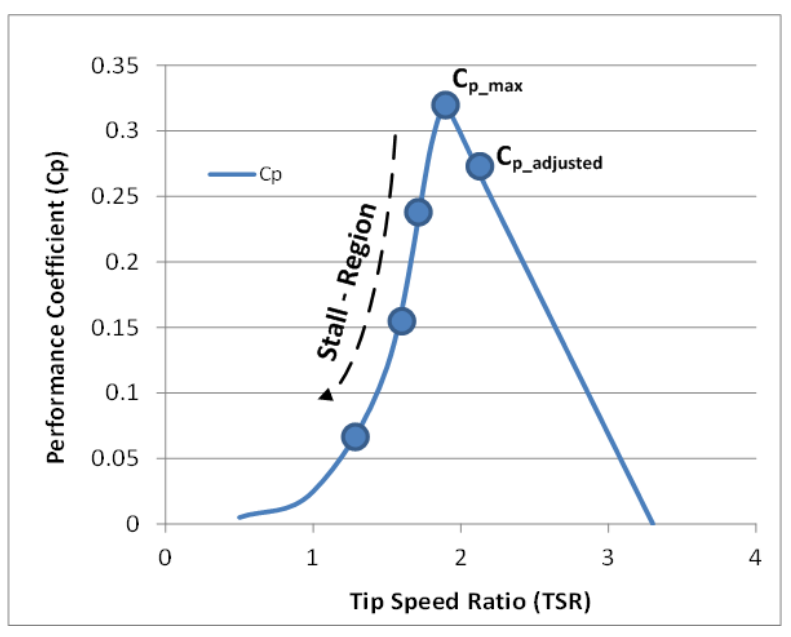

Figure 8. Illustration of the operating point for different modes of operations

A simple modification to the power equation is as follows:

$$
\begin{aligned}
& \omega_{\text {change }}=0.8 \text { p.u. } \\
& \mathrm{K}_{\text {slope }}=1.0 \\
& \text { If }\left(\omega<\omega_{\text {change }}\right) \text { then } \\
& \mathrm{K}_{\text {gain }}=0.8 \\
& \text { Else } \\
& \mathrm{K}_{\text {gain }}=\mathrm{K}_{\text {slope }}\left(\omega-\omega_{\text {change })}+0.8\right. \\
& \text { endif }
\end{aligned}
$$

where:

$\omega_{\text {change }}=$ the rotational speed when the slope is changed

$\mathrm{K}_{\text {slope }}=$ the steepness of the slope applied

Note that the parameters above can be set according to the desired operation of a particular tidal turbine and/or the local environment where the tidal turbine is installed.

$$
P_{\text {gen }}=K_{\text {gain }} K_{\text {Cpmax }} \omega^{3}
$$

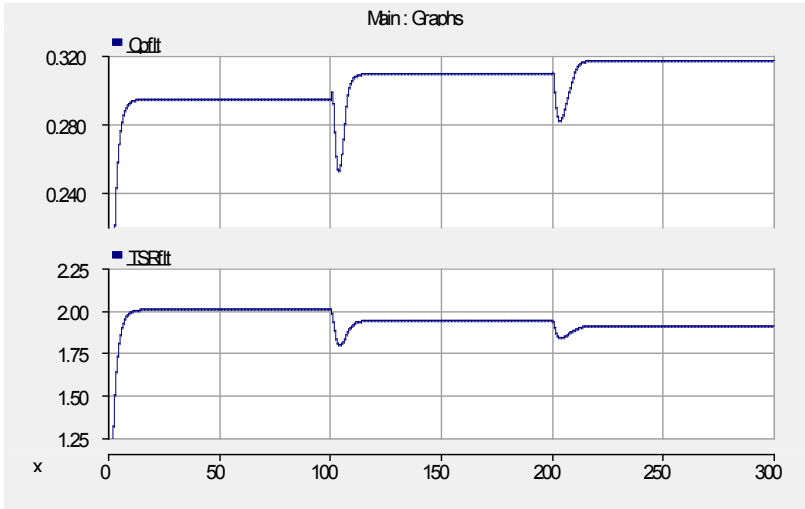

Figure 9. Performance coefficient, $\mathrm{C}_{\mathrm{p}}$, and TSR corresponding to Figure 8

Figure 9 shows the performance coefficient, $\mathrm{C}_{\mathrm{p}}$, improves and the TSR gets closer to the TSR target $\left(\mathrm{TSR}_{\text {tgt }}\right.$ where TSR $=1.9$ ), as the water flow increases from $2.2 \mathrm{~m} / \mathrm{s}$, to $2.75 \mathrm{~m} / \mathrm{s}$, to $3 \mathrm{~m} / \mathrm{s}$. Notice the improvements in both the TSR (closer to 1.9) and $\mathrm{C}_{\mathrm{p}}$ (closer to 0.318) values as the water flow increases from $2.2 \mathrm{~m} / \mathrm{s}$ to $3 \mathrm{~m} / \mathrm{s}$.

\section{B. The impact of water flow ramping}

In the previous sections, we examined an extreme condition where the water flow changes abruptly like steps. This works well to exercise the control systems implemented and to investigate the control algorithms suitable for extreme conditions. However, the changes in water flow are generally gradual. Here, we investigate different ramping rates on the water flow and observe the behavior of the turbines under these conditions. With a low ramping rate, the water flow changes at a slower rate; thus, the rotational rotor speed of the turbine is able to follow the changes of the water flow. This condition will make it possible for Point A and Point B shown in Figure 4 to stay together when the rotational speed of the turbine is able to follow the changes of the water flow.

To show the impact of the water flow ramp rate, we examine the same system with normal inertia operating with the water flow changing at 100 seconds and 200 seconds at a ramping rate of $0.1 \mathrm{~m} / \mathrm{s}$. As shown in Figure 10, if the ramping rate of the water flow is reduced, the gain factor can be increased to Kgain $=0.96$; thus, it is very close to Cpmax operation.

The corresponding Cp and TSR of the turbine are shown in Figure 11, indicating that the turbine operates at higher efficiency with the gain adjusted closer to 1.0 . Note that this is an additional $16 \%$ increase in energy conversion efficiency gained from the knowledge that the maximum ramping rate of the water flow is at $0.1 \mathrm{~m} / \mathrm{s}$. The setting of the Kgain may be adjusted for different sites for installation; this number is different from one place to another, from one time of day to the next, or from season to season depending on the nature (ramping rates) of the water flow. Perhaps an adaptive gain schedule can be implemented as we accumulate more experience and data from the field tests conducted during the coming years. 


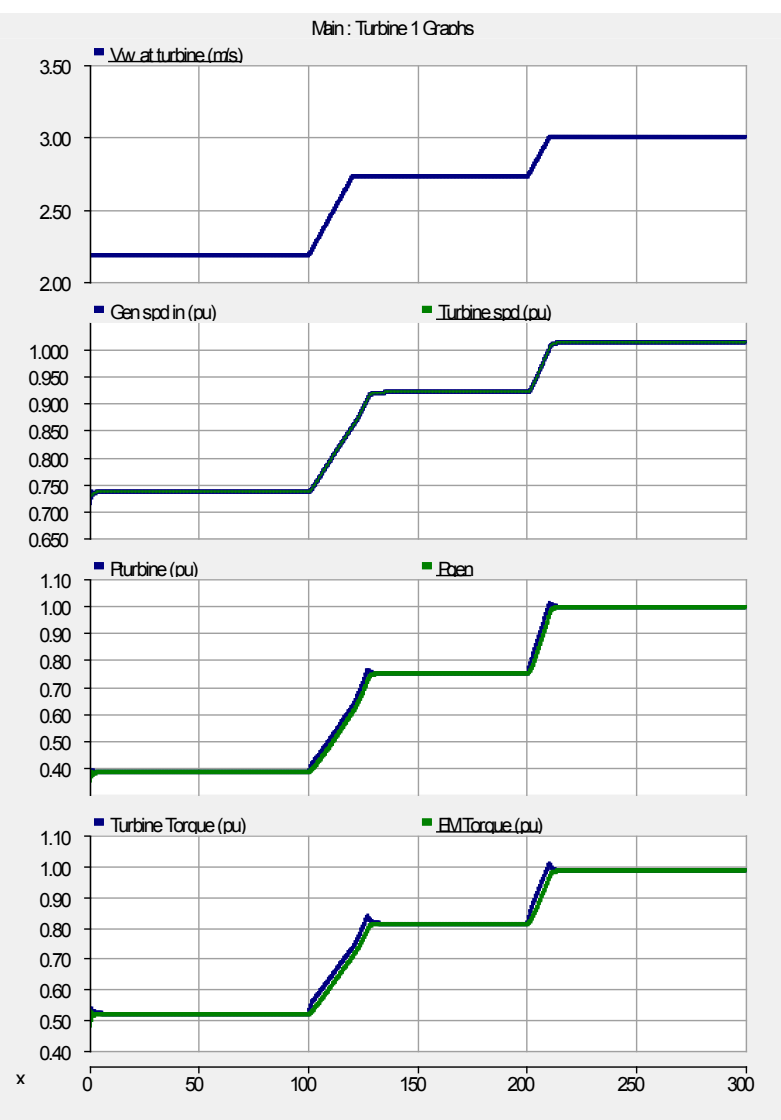

Figure 10. Kgain $=0.96, \mathrm{H}=4.94 \mathrm{~s}$, and ramp rate at $0.1 \mathrm{~m} / \mathrm{sec}$

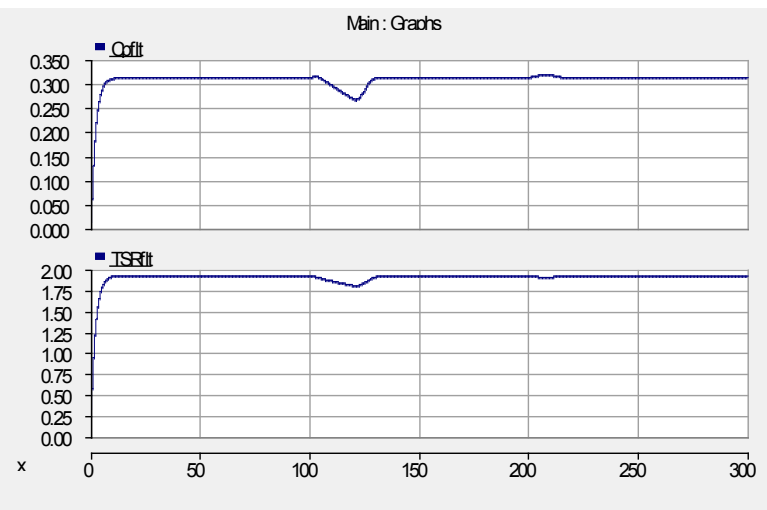

Figure 11. The corresponding Cp and TSR characteristics for the system settings shown in Figure 10.

\section{CONCLUSION}

This paper presented the basic operation of a river or tidal generator. The similarities of this type of generation system to those of a wind turbine system make it possible to start the analysis by using the basic equations used to describe wind power generation. The data analysis and calculations are given per unit when possible to make the analysis as generic as possible and to accomplish two major goals: to transport the control system to different power ratings and to make the analysis agnostic to different manufacturers; thus, no proprietary information is revealed.

- We proposed a turbine control by utilizing the rotational speed of the turbine only. This controller is very stable; however, a sudden change of water flow may stall the turbine unexpectedly. To stabilize the system, we propose two concepts: (a) derating the turbine with a fixed-gain setting throughout the water flow, resulting in a lower $\mathrm{C}_{\mathrm{p}}$ than maximum possible $\mathrm{C}_{\mathrm{pmax}}$, and (b) using adaptive gain as a function of the rotational speed to allow for higher hydrodynamic efficiency $\left(\mathrm{C}_{\mathrm{p}}\right)$ of the turbine in the region close to the rated rotor speed.

- Finally, we investigate the impact of the ramping of the water flow. Since the step change of the water flow discussed in Section III A is used to illustrate the potential of instability, in Section III B we investigate the ramping of the water flow. This is the more natural rate of change in the water flow, which reflects the nature of river or tidal generation. As shown, a gain closer to 1.0 can be used to achieve a higher operating $\mathrm{Cp}$ range, and yet, a stable operation.

- The actual implementation of the control strategy should be adapted from site to another site, possibly from season to season throughout the years. From the field experience one can adjust the strategy to increase energy capture, to optimize the electromechanical compliance, and/or to improve the overall performance of a tidal plant or cascaded river generators, as we gained experience operating the plant or generator over the years.

\section{ACKNOWLEDGMENT}

This is a collaborative work between Ocean Renewable Power Company and the National Renewable Energy Laboratory (NREL) and was supported by the U.S. Department of Energy under Contract No. DE-AC36-08GO28308 with NREL.

\section{REFERENCES}

[1] P. O. Okorie and A. Owen, "Turbulence: Characteristics and its Implications in Tidal Current Energy Device Testing," presented at OCEANS, Quebec City, Canada, Sept. 15-18, 2008.

[2] D. Li, Y. Yao, Q. Chen, and Z. Ye, "Numerical Simulation of Tidal Current Energy in Yangtze Estuary-Hangzhou Bay, China," presented at OCEANS, Genoa, Italy, May 18-21, 2005.

[3] M. Liu, W. Li, R. Billinton, C. Wang, and J. Yu, "Probabilistic Modeling of Tidal Power Generation," in Proc. 2015 IEEE Power \& Energy Society (PES) General Meeting, Denver, Colorado, July 26-30, 2015.

[4] E. Muljadi and Y. Yu, "Review of Marine Hydrokinetic Power Generation and Power Plant," Journal of Electric Power Components and Systems, vol. 43, no. 12, 2015, Special Issue: Renewable Energy Devices and Systems - State-of-the-Art and Future Trends, Taylor \& Francis publications.

Y. Jiang, M.F. Rong, and L.Y. Hua, "Variable Speed Constant Frequency Tidal Current Energy Generation and Control Strategy for Maximum Power Point Tracking and Grid Connection," presented at the International Conference on Sustainable Power Generation and Supply (SUPERGEN), Nanjing, China, April 6-7, 2009.

[5] J.S. Choi, R.G. Jeong, J.H. Shin, C.K. Kim, and Y.S. Kim, "New Control Method of Maximum Power Point Tracking for Tidal Energy Generation System," in Proc. International Conference on Electrical Machines and Systems, Seoul, Korea, Oct. 8-11, 2007. 